\title{
O papel do Processamento de Língua Natural e da Representação de Conhecimento na extração de informações em mensagens textuais na Educação a Distância*
}

\author{
Sandro J. Rigo ${ }^{1}$, Evandro M. Flores ${ }^{1}$, Márcia Abech ${ }^{1}$, Jorge L. V. Barbosa ${ }^{1}$, \\ Cristiano Costa ${ }^{1}$, Lucas L. Martins ${ }^{1}$, Thais B. Oliveira ${ }^{1}$, Denis A. Araujo ${ }^{1}$ \\ ${ }^{1}$ Programa de PósGraduação em Computação Aplicada - PIPCA - UNISINOS \\ São Leopoldo - RS - Brazil \\ \{rigo, cac, jbarbosa\}@unisinos.br, \{evandrometzflores, marcia.abech, \\ denis.andrei.araujo, lucaslaramartins, borgesoliveira.thais\}@gmail.com
}

\begin{abstract}
Particularly in distance learning, the expansion of the use of digital mediation can generate information that supports the identification of needs of students. This article presents a research about potential use of messages in text format to identify the expression of doubts. Our approach differential is the adoption of Natural language processing and Knowledge Representation resources. Evaluations carried out with actual data from monitoring groups of students indicate that the approach is promising and shows good results. Are highlighted comparative cases showing that the approach adopted allows for greater precision in the identification of events.
\end{abstract}

Resumo. A ampliação do uso de mediação digital, particularmente na Educação a Distância, pode gerar informações que apoiam a identificação de necessidades dos alunos. Este artigo apresenta uma investigação sobre o potencial de utilização das mensagens em formato textual para identificar a expressão de dúvidas. A abordagem adotada diferencia-se de outras existentes através da adoção de recursos de Processamento de Língua Natural e de Representação de Conhecimento. Avaliações realizadas com dados reais de acompanhamento de turmas de alunos indicam que a abordagem é promissora. O texto destaca casos comparativos em que a abordagem proposta permite maior precisão na identificação dos eventos.

\section{Introdução}

A atual utilização em larga escala dos recursos de mediação digital pode gerar informações de fundamental importância para a identificação e o posterior atendimento de necessidades dos alunos. Ações diversas na área de Informática na Educação vêm aprofundando estudos neste sentido. Alguns exemplos de uso dos dados gerados na mediação digital podem ser observados nas iniciativas de Mineração de Dados Educacionais (Rigo, 2012), na aplicação de recursos de Computação Afetiva (Ranilson, 2012; Iepsen, 2011) na melhora da percepção de interação dos alunos em ambientes

\footnotetext{
* Este trabalho teve apoio do Concelho Nacional de Desenvolvimento Científico e Tecnológico (CNPQ) através do Edital MCT/CNPq N. 75/2010 - RHAE Pesquisador na Empresa e da Unidade de Novos Negócios da GVDASA Informática LTDA.
} 
Virtuais. Dentre as várias possibilidades existentes em Ambientes Virtuais de Ensino e Aprendizagem (AVEAs), a análise dos dados textuais gerados durante o processo de mediação digital pode apoiar a identificação das necessidades de alunos, pois o material escrito é potencializado neste contexto (Macedo et al. 2009). Relacionado com este fator, o crescimento da adoção de modelos de Educação a Distância (EAD) e de outras modalidades voltadas para o atendimento em maior escala dos estudantes vem gerando demandas específicas, ligadas à atuação dos professores e tutores, em especial. $\mathrm{O}$ amplo volume de material textual implica na maior necessidade de tempo disponível para a sua leitura e tratamento. Este contexto é motivador para a pesquisa e desenvolvimento de formas alternativas e viáveis de tratamento automático de parte destas informações (Malin, 2009; Machado, 2010).

Este trabalho investiga possibilidades de melhorias de resultados obtidos em trabalhos na área, que abordam o problema da identificação de eventos expressos textualmente a partir de análises de expressões textuais, ferramentas de classificação textual ou de mineração textual (Oliveira e Esmin, 2012; Silva et al, 2012, Azevedo, 2011). Como relatado em (Lesmo et al, 2013), as abordagens para identificação de eventos ou informações com base apenas em aspectos textuais podem ser melhoradas nos seus aspectos de precisão e de flexibilidade quando acrescidas de camadas adicionais de informações, tais como as geradas por elementos linguísticos ou semânticos (Dedek e Vojtas, 2011; Wimalasuriya e Dou, 2010).

Dado o contexto de aplicação relevante para apoio na educação e a ampliação crescente do conjunto de dados disponíveis, este trabalho apresenta e analisa uma abordagem de extração de informações destinada a localizar dificuldades expressas textualmente pelos alunos em AVEAs, com base na integração de Processamento de Língua Natural (PLN) e de representação de conhecimento. Os recursos de PLN ampliam o conjunto de dados disponíveis para a análise e identificação das informações textuais. A utilização de representação de conhecimento possibilita estabelecer conexões entre os assuntos e situações descritas e eventuais materiais de apoio, tais como os materiais instrucionais ou objetos de aprendizagem. Para avaliar esta abordagem foi realizado um ciclo de estudos com material gerado em um AVEA, através do uso da análise textual de postagens realizadas pelos alunos. Na primeira etapa não foram utilizados recursos linguísticos e semânticos, para proporcionar um contexto de comparação com a segunda etapa, em que foram aplicadas análises com uso destes recursos. Foram realizadas, por especialistas, validações manuais dos resultados obtidos e identificados pontos em que a abordagem empregando recursos linguísticos e semânticos apresenta melhorias quanto aos resultados obtidos inicialmente.

\section{Abordagem adotada}

Este trabalho visa utilizar técnicas de extração de informações para a identificação de mensagens de textos expressando dúvidas de alunos e posteriormente a identificação dos tópicos associados com estas dúvidas. A partir desta identificação, estes resultados podem ser utilizados para apoio na atuação dos professores no contexto de cursos na modalidade de Educação a Distância, ou para a localização de material instrucional relacionado com o assunto mencionado. Estas duas possibilidades, entretanto, não são tratadas em detalhes neste trabalho, sendo sugeridas como possibilidades de utilização futura. $\mathrm{O}$ texto a seguir é dedicado à descrição e análise da abordagem de integração 
entre recursos linguísticos proporcionados com o uso de recursos de PLN (Lidy, 2003) e recursos semânticos obtidos com o uso de representação de conhecimento em Ontologias (Guarino, 2009). A figura 1 resume os componentes gerais utilizados na abordagem adotada. A primeira etapa envolve o acesso aos dados gerados em um AVEA, sendo que esta abordagem pode ser utilizada com diferentes AVEAs. O processo de Seleção de Dados é utilizado para a escolha e tratamento prévio dos conjuntos de dados textuais desejados. Já o processo de Tratamento Textual utiliza o apoio do sistema Palavras (Bick, 2000), que é o analisador morfológico e gramatical utilizado. O processo de Conversão de formato manipula os dados obtidos no processo anterior e gera elementos de uma ontologia com cada uma das frases analisadas, segundo o padrão de anotação POWLA (Chiarcos, 2012). Isso possibilita, no processo seguinte, a utilização integrada de regras linguísticas e de elementos de ontologias de domínio para a identificação dos eventos desejados, ou seja, as dúvidas e assuntos relacionados.

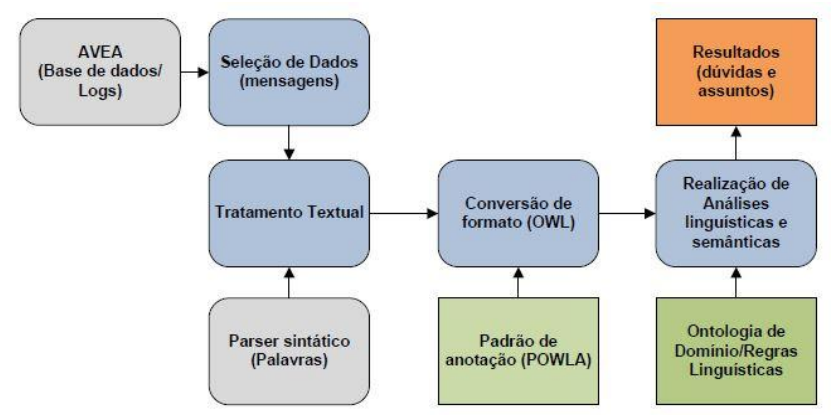

Figura 1. Visão geral dos componentes e processos

A seguir são detalhados pontos importantes da abordagem adotada. As frases obtidas no processo de Seleção de dados são tipicamente expressões de dúvidas de alunos encontrados nos mais diversos formatos, devido ao uso da linguagem natural nos fóruns de discussão. Entre as frases podem ser encontradas referências a materiais dentro do contexto do AVEA, expressões de dúvidas ou citações de conceitos das matérias estudadas, entre outras. No exemplo listado abaixo, na figura 2, é citada uma página do livro texto utilizado ("Na página 16..."), uma expressão denotando dúvida (“... não consegui compreender...") e conceitos da área de linguagem de programação (“..intervalo de valores.... tipos de dados primitivos...").

Na página 16 , não consegui compreender o intervalo de valores dos tipos de dados
primitivos da linguagem java...Qual a relação do identificador e espaço de
armazenamento com aquela informação de intervalo?

Figura 2. Exemplo de mensagem utilizada

O processo de Tratamento textual destas frases com o analisador morfológico e gramatical permite que sejam geradas informações detalhadas para cada uma delas, organizadas em uma estrutura em que as palavras são classificadas de acordo com sua função na frase. Além disso, podem ser identificados os fragmentos textuais que formam cada frase. Este resultado é gerado em um arquivo em formato XML, o que facilita a sua manipulação posterior. Uma versão resumida deste resultado é exibida na figura 3, onde pode ser observada a frase original (no elemento " $s$ ", atributo "text"), a análise de cada palavra (nos elementos "terminals") e análises de componentes das 
frases (nos elementos "nonterminals") ${ }^{1}$. A identificação de eventos de interesse pode ser realizada a partir deste conjunto ampliado de informações, proporcionando maior flexibilidade e amplitude na geração de regras que possibilitem o relacionamento destes elementos em diversos níveis. Por exemplo, podem ser diferenciados os usos de palavras como verbo ou substantivo, analisados os seus modificadores ou ainda relacionados os antecessores de uma determinada expressão.

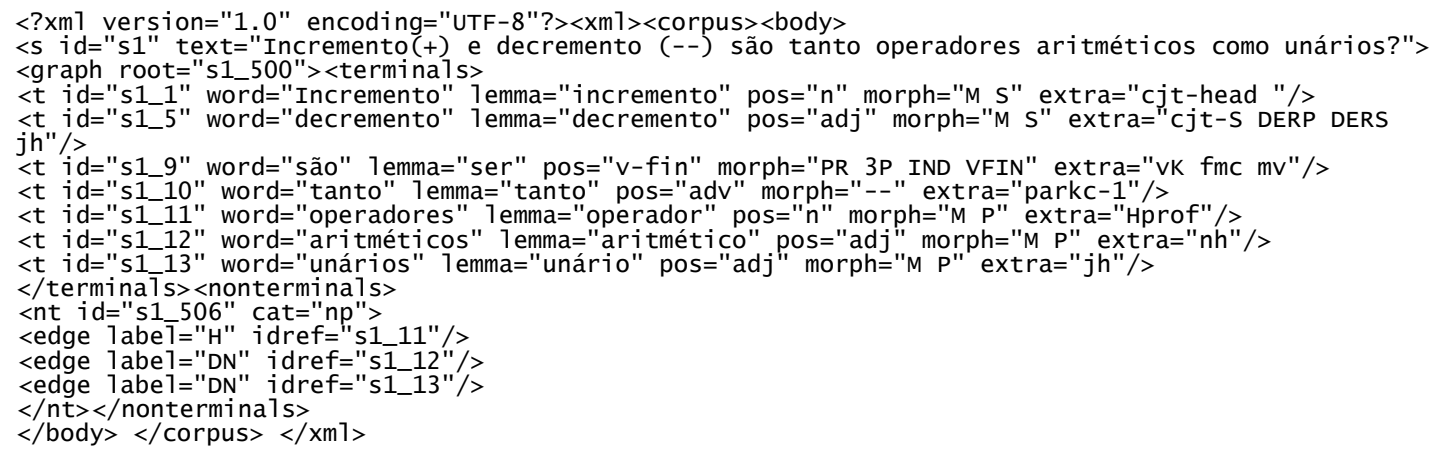

Figura 3. Exemplo de resultados da análise morfológica e gramatical

Para que possam ser incluídas neste contexto as informações semânticas, descritas em ontologias de domínio e voltadas para as diferentes matérias em análise em cada turma, foi utilizada nesta abordagem um modelo de anotação de dados linguísticos. O padrão utilizado foi o POWLA (Chiarcos, 2012), que possibilita a transformação das informações obtidas com o analisador morfológico para a linguagem $\mathrm{OWL}^{2}$. Como resultado as frases a serem analisadas estarão disponíveis com uma anotação rica em detalhes, em uma ontologia descrita com a linguagem OWL.

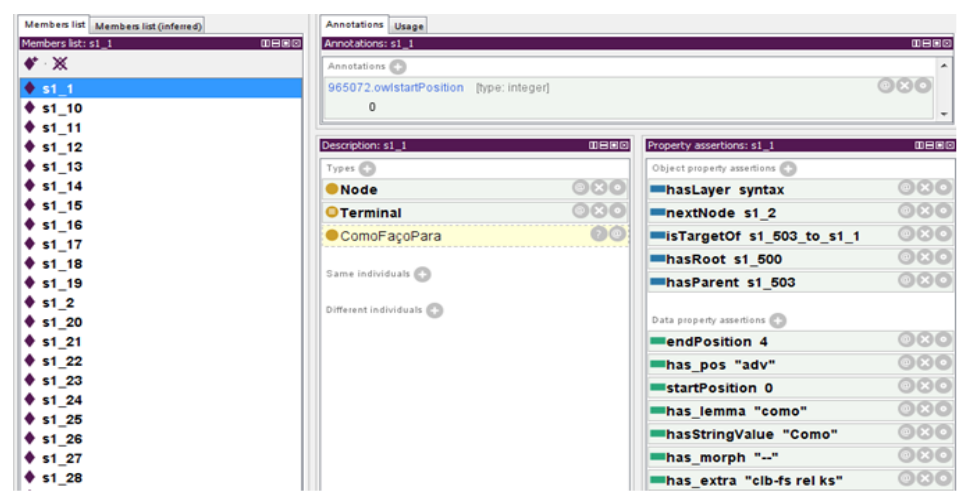

Figura 4. Visualização de padrão POWLA e representação de frases

A figura 4 mostra uma tela do editor de ontologias Protége ${ }^{3}$, com a representação de elementos textuais analisados e representados a partir do padrão POWLA. Nesta figura pode ser identificado, na aba "properties assertion", um conjunto de elementos gerados pelo analisador morfológico, representado como um conjunto de relações para determinado elemento textual. Por exemplo, o item "S1_1" está associado com as propriedades "has_pos", "has_lemma", "has_extra", "hasParent", "nextNode" e "hasRoot". Estas propriedades podem ser utilizadas na criação de regras de

\footnotetext{
${ }^{1}$ Maiores detalhes sobre o formato em uso podem ser obtidos em (Bick, 2000).

${ }^{2}$ http://www.w3.org/2001/sw/wiki/OWL

3 http://protege.stanford.edu/
} 
relacionamento e identificação de eventos. Um dos resultados desse processo é a possibilidade de utilização de mecanismos de inferência para a integração das regras de identificação e eventos com os elementos disponibilizados pelo padrão POWLA, utilizando a expressividade disponível na linguagem OWL. Assim é possível também a integração de ontologias de domínio contendo os conceitos tratados em matérias sendo analisadas.

A figura 5 apresenta um exemplo de regra em Semantic Web Rule Language $\left(\mathrm{SWRL}^{4}\right)$ para identificar um contexto de uma frase contendo a expressão de uma dúvida, o que é feito utilizando a anotação dos elementos das frases em OWL, com o padrão POWLA. Por sua vez, a integração com uma ontologia de domínio permite que sejam utilizadas regras relacionando os conceitos existentes na ontologia com os elementos textuais. Desta forma pode ser modelado em regras o relacionamento de uma estrutura de frase e um determinado conceito, ou então podem ser utilizadas as hierarquias e relações da própria ontologia de domínio para a identificação de assuntos mais específicos ou mais abrangentes relacionados com o assunto identificado.

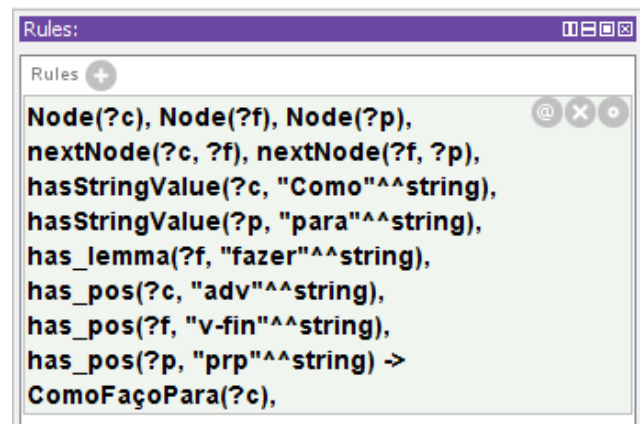

Figura 5. Regra em SWRL com representação linguística de elementos

Por fim, cabe destacar os seguintes diferenciais da abordagem adotada. Em primeiro lugar, trata-se de um processo flexível, que pode ser utilizado em diferentes AVEAs e com diferentes finalidades. A utilização de um analisador morfológico e gramatical permite que sejam utilizadas informações adicionais, de modo a ampliar a possibilidade de uso de regras e padrões de identificação de eventos. Já a adoção do padrão de anotação de dados POWLA e a representação das informações em padrão OWL possibilita um aumento na flexibilidade de tratamento das informações, pois habilita o uso de regras em padrão SWRL e, além disso, permite a inclusão de elementos descritos em outras ontologias de domínio para composição das regras.

A análise e a execução de regras são determinadas com base em um mecanismo de inferência, tendo sido desenvolvida para validar esta abordagem uma aplicação em linguagem de programação $\mathrm{Java}^{5}$, com base na biblioteca OWL $\mathrm{API}^{6}$, utilizando o mecanismo de inferência Pelet ${ }^{7}$. O resultado final da execução das regras é gerado em uma base de conhecimento contendo os eventos identificados, podendo essa base ser

\footnotetext{
${ }^{4}$ http://www.w3.org/Submission/SWRL/

5 http://docs.oracle.com/javase/

${ }^{6} \mathrm{http}: / /$ owlapi.sourceforge.net/

http://clarkparsia.com/pellet/
} 
posteriormente utilizada para atividades de visualização ou de relacionamento com outros conjuntos de dados do AVEA.

\section{Experimentos e validações}

Para os experimentos descritos nesta seção, foram utilizados dados obtidos em contexto real de atividades acadêmicas em cursos na modalidade de EAD, em atividade acadêmica voltada para o tema de conceitos básicos em Programação de Sistemas. Para as avaliações foram utilizadas mensagens postadas em fóruns de dúvidas, tendo sido utilizado um total de 1.195 postagens geradas ao longo de um bimestre de aulas. O AVEA utilizado para esta coleta foi o MOODLE ${ }^{8}$.

A metodologia adotada para os experimentos foi composta por duas etapas. $\mathrm{Na}$ primeira etapa a identificação de eventos não empregou as informações linguísticas e semânticas, mas um léxico composto a partir da análise da amostra textual utilizada. Os resultados desta etapa foram avaliados por um especialista em linguística e um especialista no domínio de conhecimento e destacadas, além das identificações corretas, aquelas nas quais a falta de informações semânticas ou linguísticas pode ter interferido no resultado, gerando um resultado incompleto ou incorreto. Um exemplo deste tipo de situação pode ser visto na figura 6. No item "1" a análise sem informações linguísticas identificou o termo "for", que é parte das palavras reservadas do assunto sendo estudado, como sendo um elemento da dúvida expressa na frase. Porém neste caso o termo "for" é parte da frase e não denota uma dúvida. Já na segunda, os termos "Array" e "ArrayList" são identificados, pois fazem parte do conjunto de conceitos tratados, porém a frase não está expressando uma dúvida e sim uma afirmação do aluno no sentido contrário.

1) Professora, Fiquei meio confuso com a questão... o método " leumaIdade() " não finaliza enquanto a idade não for iqual 0 (idade $!=0$ ), ...

2) Agora entendi como resolver o problema de outra forma. Bem, acabei aprendendo a usar o Array e o ArrayList.

Figura 6. Exemplos de análises com identificação parcialmente correta

Na segunda etapa da avaliação foram utilizadas as situações identificadas como problemáticas a partir do primeiro estudo e com isso geradas regras de identificação de eventos com base na estrutura linguística das frases e com uso da ontologia de domínio. Um exemplo deste tipo de regra pode ser visto na figura 5, que identifica a utilização de um verbo ("fazer"), antecedido de um advérbio ("como") e seguido de uma preposição, o que garante que o elemento seguinte na frase, que pode ser identificado com um fragmento da sentença ou com um termo simples, estará relacionado corretamente com expressão de dúvida indicada. A partir desta modelagem de regras o sistema foi utilizado para uma nova etapa de avaliações das frases, nas quais foram identificadas melhorias na precisão de identificação dos eventos.

\subsection{Detalhamento de experimentos}

Objetivando o tratamento inicial das informações textuais, foi realizada, por um especialista em linguística e por um especialista no domínio de conhecimento, uma análise manual e individual de uma parte das postagens de mensagens em fóruns dos

\footnotetext{
${ }^{8}$ http://www.moodle.org
} 
alunos das turmas envolvidas no estudo de caso. Para definir o que seria uma mensagem indicadora de dúvida, os especialistas optaram por criar um critério de seleção incluindo mensagens que apresentam um questionamento sobre determinado assunto da atividade acadêmica ou qualquer dúvida relacionada ao andamento da matéria. Com este critério foi criado um léxico de palavras definidoras de mensagens com temas relacionados a dúvidas. Neste estudo foram analisadas metade das 1.195 postagens e foram realizados levantamentos das palavras mais comuns naquelas mensagens que, segundo os especialistas, apresentavam o tema de dúvidas. Com este léxico, composto por 53 termos, foi desenvolvido um filtro para primeira etapa de validação, utilizado na identificação, nas mensagens do grupo restante, dos termos constantes no léxico.

Para identificar qual o assunto da dúvida, foi construída uma ontologia que representa a estrutura de conceitos relacionados ao domínio de conhecimento em estudo. Essa ontologia está descrita na figura 7 , nos seus componentes principais. A ontologia foi descrita para prover mecanismos para a estruturação de conceitos e suas relações, bem como para facilitar a representação textual destes, além da identificação de sinônimos e termos compostos. Esta identificação é um dos principais desafios do tratamento de mensagens em linguagem natural. Para sua construção foram observadas metodologias conhecidas (Staab e Studer, 200; Noy e McGuiness, 2001) e consultados especialistas no assunto específico. O modelo utilizado representa a atividade acadêmica em seus assuntos, portanto composta de vários conceitos, que por sua vez podem possuir conceitos associados. Cada conceito possui vários termos que podem ser utilizados na expressão textual dos questionamentos dos estudantes. A ontologia desenvolvida e utilizada no estudo de caso de avaliação é composta por 40 conceitos organizados em 5 classes principais.

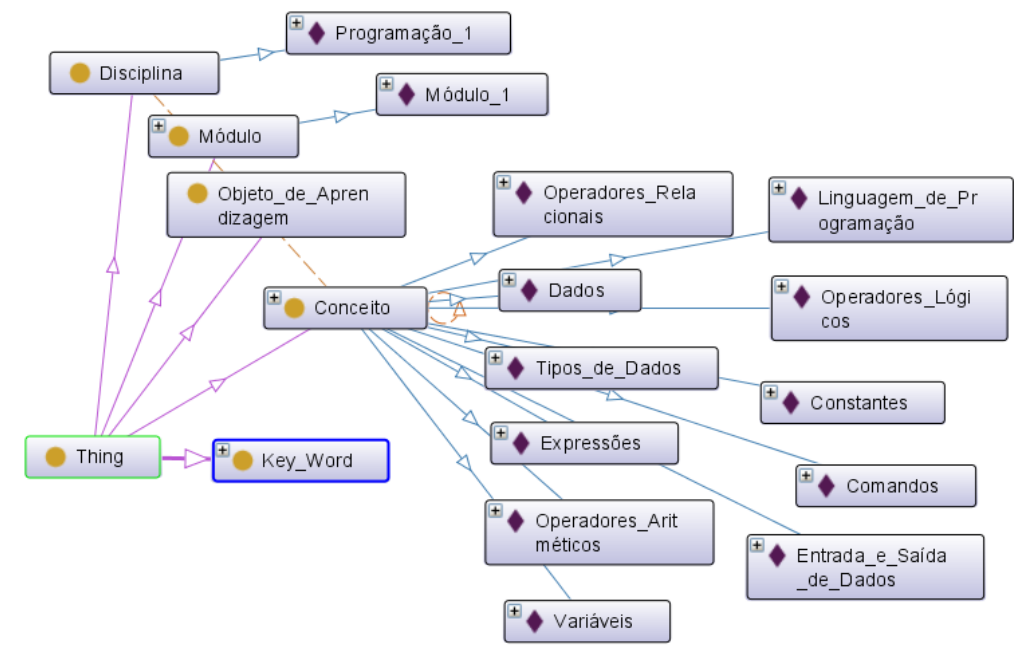

Figura 7. Visão parcial de ontologia de domínio utilizada

O uso desta ontologia viabiliza um sistema de busca e comparação onde cada termo, dentro de cada postagem textual, é pesquisado no léxico e na ontologia. A análise manual, feita pelos especialistas envolvidos, dos resultados obtidos permitiu identificar situações nas quais a utilização direta dos elementos textuais, tanto representando termos contendo dúvidas, como contendo a descrição dos assuntos tratados, não permitiu a geração de um resultado correto ou completo. Uma parte do conjunto total de mensagens utilizadas na análise inicial foi empregada em uma verificação com o filtro 
composto pelo léxico criado e pela ontologia de domínio descrita. Este conjunto de 594 mensagens foi utilizado para o teste de identificação de dúvidas e de conceitos, sendo comparados os resultados obtidos automaticamente com a identificação manual das mensagens. Neste caso, em um primeiro resultado, foi obtida uma taxa de acerto de $79,3 \%$, sendo que este valor pode ser associado com dois aspectos. O primeiro é a falta de elementos textuais para a identificação de todas as ocorrências, o que pode ser superado com o aumento dos dados no léxico e na ontologia. O segundo é a diversidade de formatos das mensagens em linguagem natural, que permite que interpretações inexatas ocorram.

Para avaliar as possibilidades de ampliação da taxa de identificação correta das informações, foi realizada uma análise nas mensagens onde foram detectadas incorreções, sendo que a análise manual realizada pelos especialistas permitiu identificar um conjunto de 17 destas situações que foram selecionadas para a descrição de regras para o tratamento com informações semânticas e linguísticas. Algumas destas situações são exemplificadas na figura 6. Para a descrição de regras que permitam restringir as situações com descrição de erros, foram empregados os elementos que compõem o diferencial do trabalho, ou seja, os elementos descritos na análise sintática das frases com o analisador morfológico e ainda a descrição dos conceitos na ontologia de domínio. Um exemplo deste tipo de regra está descrito na figura 5. Com o tratamento posterior, com base nas regras descritas e uso dos recursos linguísticos e semânticos, as frases destacadas foram corretamente identificadas em seus eventos, de acordo com a aplicação das regras construídas.

Um dos pontos importantes desta abordagem é a possibilidade de contar com uma análise morfológica, de modo a não haver incorreções na identificação dos elementos que complementam as expressões de dúvidas. Desta forma é possível, com a correta descrição de regras de identificação, a identificação com precisão de situações não detectadas apenas com o uso de informações textuais. Além deste aspecto, a ontologia de domínio utilizada serve como apoio na localização de relacionamentos de composição e de dependência entre conceitos, o que vem apoiar as atividades de identificação de assuntos e temas complementares ou de requisitos para assuntos sendo estudados por alunos.

\section{Trabalhos relacionados}

Diversos trabalhos vêm sendo desenvolvidos para o tratamento de dados textuais e identificação de eventos e informações. Em alguns casos, trata-se da utilização de ferramentas estatísticas, algoritmos de casamento de padrões ou de aprendizagem de máquina para identificação de elementos textuais importantes e apoio à produção textual (Macedo et al, 2009; Klemann et al, 2009, Azevedo 2011; Silva et al, 2012), sendo que nestes casos os resultados obtidos são por vezes limitados aos conjuntos de padrões utilizados ou aos limites de tratamento de informações apenas textuais.

Voltado para estudos com opiniões de consumidores, são conhecidos trabalhos com base no apoio de técnicas de pré-processamento que possibilitam o tratamento de stop-words e stemmer, entre outras características (Pang et al, 2002), seguidos por etapas de classificação com uma grande variação de técnicas oriundas da área de 
Inteligência artificial (Chen, 2011; Tang, 2009), sendo que algumas abordagens adotam o uso de léxicos descrevendo a polaridade de termos encontrados em textos (Liu, 2012).

O trabalho aqui apresentado difere de outras abordagens pelo fato de explorar um conjunto mais amplo de informações, compostas por informações linguísticas e semânticas, o que pode ser traduzido em um arcabouço mais robusto para a identificação de padrões tão diversificados como os encontrados na linguagem natural escrita.

\section{Considerações Finais}

Este artigo apresentou a integração de recursos de PLN e Representação de conhecimento para a identificação de eventos em mensagens textuais geradas no contexto de cursos em Educação a Distância. Foi realizada a coleta e tratamento de amostra textual de mensagens de fóruns e a geração de um léxico e de uma ontologia de domínio para identificação de eventos expressando dúvidas e conceitos tratados. Estes resultados foram analisados por especialistas e determinadas situações de identificação incorreta ou incompleta, que foram utilizadas como base para a geração de regras utilizadas a partir de informações linguísticas e semânticas para a identificação mais precisa dos eventos.

Como possibilidade de trabalhos futuros, está sendo estudada a possibilidade de encadeamento e de relacionamento de conceitos descritos na ontologia, de forma a localizar assuntos mais abrangentes e/ou mais específicos relacionados com os conceitos citados, de modo a apoiar a identificação do nível de conhecimento sobre os conceitos tratados.

\section{Referências}

Azevedo, B. F. T. (2011) MINERAFÓRUM: Um recurso de apoio para análise qualitativa em fóruns de discussão. Programa de Pós-Graduação em Informática na Educação. UFRGS. Tese de Doutorado.

Bick, E. (2000) "The Parsing System PALAVRAS: Automatic Gramatical Analysis of Portuguese in a Constraint Grammar Framework". Århus University. Århus: Århus University Press.

Bercht, M.. (2001) Em Direção a Agentes Pedagógicos com Dimensões Afetivas. Instituto de Informática. UFRGS. Tese de Doutorado.

Chen, L. S.; Liu, C. H.; Chiu, H. J. A neural network based approach for sentiment classification in the blogosphere. Journal of Informetrics, [S.1], v.5, n.2, p.313-322, 2011.

Chiarcos, Christian. POWLA: Modeling linguistic corpus in OWL/DL. Proceedings of 9th Extended Semantic Web Conference (ESWC2012), 2012a.

Desmarais, M.C., Baker, R.S.J.d. (2012) A Review of Recent Advances in Learner and Skill Modeling in Intelligent Learning Environments. User Modeling and UserAdapted Interaction, v.22, n.1-2, p.9-38.

Dedek, J.; Vojtas, P. Semantic Annotation Semantically: Using a Shareable Extraction Ontology and a Reasoner. In: Proceedings of SEMAPRO 2011, The Fifth International Conference on Advances in Semantic Processing, p. 29-34, Lisbon, 2011.

Guarino, N., What is an Ontology?. Handbook of ontologies. Second Edition. Springer 
Verlang-Berlin.2009, p. 1-17.

Iepsen, E. F., Bercht, M., Reategui, E. 2011, Detecção e Tratamento do Estado Afetivo Frustração do Aluno na Disciplina de Algoritmos. Anais do XXII SBIE Aracaju. 2011.

Klenmann, M.; Lorenzatti, A.; Reategui, E. O Emprego da Ferramenta de Mineração de Textos SOBEK como Apoio à Produção Textual. In: SBIE, 2009, Florianópolis. Anais do SBIE, 2009.

Lesmo, L.; Mazzei, A.; Palmirani, M.; Radicioni, D.; Tulsi, P.: an NLP system for extracting legal modificatory provisions. Artificial Intelligence and Law. v. 21, p. 139-172. Springer Netherlands. 2013.

Liddy, Elizabeth D. Natural Language Processing. In: Encyclopedia of Library and Information Science, 2nd ed. New York: Marcel Decker, Inc., 2003.

Liu, B. Sentiment Analysis and Opinion Mining. Morgan and Claypool Publishers, 2012. 165p.

Macedo, A. L.; Reategui, E.; Lorenzatti, A.; Behar, P. Using Text-Mining to Support the Evaluation of Texts Produced Collaboratively. Education and Technology for a Better World, IFIP Advances in Information and Communication Technology, Volume 302. ISBN 978-3-642-03114-4. Springer Berlin Heidelberg, 2009, p. 368.

Malin, J.T., et al.Linguistic text mining for problem reports. Systems, Man and Cybernetics, 2009. SMC 2009. IEEE International Conf. San Antonio, 2009.

Machado, A. P., Ferreira, R., Bittencourt, I. I., Elias, E., Brito, P., Costa, E., Mineração de texto em redes sociais aplicada à educação a distância, Colabor@ - Revista Digital da CVA - Ricesu, ISSN 1519-8529. V.6(23), Julho de 2010.

Noy, N. F., Mcguinness, D. L., Ontology development 101 A guide to creating your first ontology, Tech. rep., Stanford Knowledge Systems Laboratory (2001).

Oliveira, R. L. J., Esmin, A. A. A. 2012, Monitoramento Automático de Mensagens de Fóruns de Discussão Usando Técnica de Classificação de Texto SemiSupervisionado. Anais do $23^{\circ}$ Simpósio Brasileiro de Informática na Educação (SBIE 2012), ISSN 2316-6533 Rio de Janeiro, 26-30 de Novembro de 2012

Pang, B. et al. Thumbs up? Sentiment Classification using Machine Learning Techniques. In: Conference on empirical methods in natural language processing. 2002. Proceedings...[S.1.:s.n.], 2002, p.78-86.

Ranilson, P, et. al. Mineração de Dados e a Gestão Inteligente da Aprendizagem: desafios e direcionamentos. CSBC, I Workshop de Desafios da Computação Aplicada à Educação. DesafiE 2012.

Rigo, S. J. ; Cazella, S. ; Cambruzzi, W. L. Minerando Dados Educacionais com foco na evasão escolar: oportunidades, desafios e necessidades. DEsafIE, Curitiba, 2012.

Silva, J. K.K., Bastos, H. P. P., Bercht, M., Wives, L. K. 2012. Automatização do Processo de Identificação de Presença Social em Fóruns e Chats . Anais do $23^{\circ}$ (SBIE 2012, ISSN 2316-6533 Rio de Janeiro, 26-30 de Novembro de 2012.

Staab, S., Studer, R. Knowledge processes and ontologies, Intelligent Systems, IEEE.2000.

Tang, H. TAN, S.; CHENG, X. A survey on sentiment detection of reviews. Expert Systems Applications. NY, USA, p.10760-10773, September, 2009.

Wimalasuriya, D. C.; DOU, D., Ontology-based information extraction: an introduction and a survey of current approaches. Journal of Information Science, v. 36, n. 3, p. 306-323, 2010. 